\title{
Formation of binary ion clusters from polar vapours: effect of the dipole-charge interaction
}

\author{
A. B. Nadykto and F. Yu \\ Atmospheric Sciences Research Center, State University of New York at Albany, 251 Fuller Road, Albany, NY 12203, USA
}

Received: 11 July 2003 - Published in Atmos. Chem. Phys. Discuss.: 2 October 2003

Revised: 9 February 2004 - Accepted: 15 February 2004 - Published: 27 February 2004

\begin{abstract}
Formation of binary cluster ions from polar vapours is considered. The effect of vapour polarity on the size and composition of the critical clusters is investigated theoretically and a corrected version of classical KelvinThomson theory of binary ion-induced nucleation is derived. The model predictions of the derived theory are compared to the results given by classical binary homogeneous nucleation theory and ion-induced nucleation theory. The calculations are performed in wide range of the ambient conditions for a system composed of sulfuric acid and water vapour. It is shown that dipole-charge interaction significantly decreases the size of the critical clusters, especially under the atmospheric conditions when the size of critical clusters is predicted to be small.
\end{abstract}

\section{Introduction}

Formation of ultrafine aerosols has received increasing attention in the last few decades due to its importance for atmospheric physics and chemistry, chemical technology and health research. The possible role of air ions in aerosol formation, which was intensively studied during seventies and earlier eighties (e.g. Mohnen, 1971; Castleman et al., 1978; Arnold, 1980; Hamill et al., 1982), has received renewed attention in recent years (e.g. Yu and Turco, 2000; Carslaw et al., 2002; Eichkorn et al., 2002; Yu, 2002, 2003). Classical theory of ion-induced nucleation (IIN) (e.g. Hamill et al., 1982; Raes et al., 1986; Laakso et al., 2002) treats the cluster formation using capillary approximation and it accounts for the charge effect on the pressure in the condensed phase only. The classical IIN model is derived assuming a flat monomer concentration profile in the vicinity of the nucleating cluster, which is a good approximation for non-polar vapours. How-

Correspondence to: A. B. Nadykto

(alexn@asrc.cestm.albany.edu) ever, the interaction of polar monomers with the electrical field of the charged particle leads to the enhancement in the monomer concentration near the particle surface (Korshunov, 1980; Nadykto et al., 2003). Recent studies (Nadykto et al., 2003) showed that the interaction of polar vapour molecules with the electrical field of charged particles may be important for the formation of small ion clusters. Nadykto et al. (2003) considered the ion-induced formation of singlecomponent particle and they concluded that the contribution of the dipole-charge attraction potential to the size of the ion clusters is significant, when polar vapours are involved in the nucleation process. In addition to the reduction of evaporation, the dipole-charge interaction enhances the condensation rate through enlargement of the effective collision cross section (Nadykto and $\mathrm{Yu}, 2003$ ). There is a clear difference between the effect due to the dipole-charge interaction in the gas phase and the Thomson effect. The Thomson effect relates to the properties of the condensed phase while the dipole-charge interaction modifies the chemical potential of the condensing monomers in the electrical field of the charged particle/cluster.

The purpose of this paper is to study the effect of dipolecharge interaction on the formation of binary cluster ions. We will derive the generalized Kelvin-Thomson equation accounting for vapour polarity, calculate the critical size of binary sulfuric acid-water ion clusters and compare our model predictions with the results of the earlier theories.

\section{Model}

In the classical binary IIN theory (e.g. Hamill et al., 1982; Raes et al., 1986; Laakso et al., 2002) in the prevailing temperature and vapor pressures of two condensing components $a$ and $b$, the critical size of binary cluster ion is determined 
from the Kelvin-Thomson equation (e.g. Laakso et al., 2002),

$\ln S^{\prime}=\frac{\bar{V}}{k T}\left[\frac{4 \sigma}{D_{p}^{\prime}}-\left(\frac{1}{\varepsilon_{g}}-\frac{1}{\varepsilon_{r}}\right) \frac{\left(q e_{0}\right)^{2}}{2 \pi^{2} \varepsilon_{0} D_{p}^{\prime 4}}\right]$,

where $S^{\prime}=S_{a}^{1-X_{b}} S_{b}^{X_{b}}, \bar{V}=\left(1-X_{b}\right) v_{a}+X_{b} v_{b}, \frac{\pi D_{p}^{\prime 3}}{6} \rho=$ $n_{a} m_{a}+n_{b} m_{b}$. Here $S^{\prime}$ is the generalized saturation ratio, $S_{a}$ and $S_{b}$ are the saturation ratios for component $a$ and $b$ respectively, $\bar{V}$ is the average molecular volume, $v_{a}$ and $v_{b}$ are the partial molecular volumes for components $a$ and $b$ respectively, $n_{a}$ and $n_{b}$ are the number of molecules in the particle for components $a$ and $b$ respectively, $m_{a}$ and $m_{b}$ are the molecule mass of components $a$ and $b$ respectively, $q$ is the number of the charges in the cluster, $e_{0}$ is the elementary charge, $\sigma$ is the surface tension, $\varepsilon_{r}$ is the relative permittivity of particle, $\varepsilon_{g}$ is the relative permittivity of the condensable vapour, $\varepsilon_{0}$ is the vacuum permittivity, $k$ is the Boltzman constant, $\rho$ is the particle/cluster density, $X_{a}$ and $X_{b}$ are molar fractions for components $a$ and $b$ respectively, and $D_{p}^{\prime}$ is the diameter of the cluster. The composition of the charged particle is decided by the following equation

$\frac{v_{a}}{\Delta \mu_{a}}=\frac{v_{b}}{\Delta \mu_{b}}$,

where $\Delta \mu_{i}=\mu_{i L}-\mu_{i g}$ is the chemical potential change from gas phase $\left(\mu_{i g}\right)$ to the condensed /liquid phase $\left(\mu_{i L}\right)$ of component $i(i=a, b)$.

In a binary system, change in the Gibbs free energy can be expressed by the following equation

$\Delta G=\Delta \mu_{a} n_{a}+\Delta \mu_{b} n_{b}+\sigma A+\frac{q^{2} e_{o}^{2}}{8 \pi \varepsilon_{0}}\left(\frac{1}{\varepsilon_{g}}-\frac{1}{\varepsilon_{r}}\right)\left(\frac{1}{r}-\frac{1}{r_{0}}\right)(3)$

where $r$ is the cluster radius, $A$ is the cluster surface area, and $r_{0}$ is the radius of the core ion. In the case of non-polar vapours we get, assuming flat vapour profile in the vicinity of the cluster nucleating, the following conventional equation

$\Delta G_{=}\left(\mu_{a L}-\mu_{a \infty}\right) n_{a}+\left(\mu_{b L}-\mu_{b \infty}\right) n_{b}+\sigma A$

$+\frac{q^{2} e_{o}^{2}}{8 \pi \varepsilon_{0}}\left(\frac{1}{\varepsilon_{g}}-\frac{1}{\varepsilon_{r}}\right)\left(\frac{1}{r}-\frac{1}{r_{0}}\right)$,

The change in the Gibbs free energy due to the phase transition relates to the difference in the chemical potentials of the gas phase molecules located near the interphase boundary (over the particle surface) and molecules in the condensed phase. Since the isothermal chemical potential of the vapour is a function of the vapour pressure only, the correction to the chemical potential is derived through the calculation of the vapour pressure of over the charged particle surface (Korshunov, 1980; Nadykto et al., 2003). The electrical field of the cluster/particle attracts polar monomers and, thus, their concentration in the vicinity of the nucleating particle and the vapour pressure over the particle surface rises. This may modify the chemical potential of the vapour molecules and change in the Gibbs free energy significantly. In the electrical field of the charged cluster/particle, the difference between chemical potentials of the polar molecules in the condensed phase and in the gas phase is given by (e.g. Nadykto et al., 2003)

$\mu_{i L}-\mu_{i g}=-k T\left[\ln \left(\frac{A_{i g \infty}}{A_{i L}}\right)+\ln \left(\frac{p_{i g}}{p_{i \infty}}\right)\right]$.

where $A_{i L}$ is activity of component $i$ in the condensed phase, $A_{i g \infty}$ is activity of component $i$ in the gas phase, $p_{i g}$ is the vapour pressure over the particle surface, $p_{i \infty}$ is the ambient vapour pressure. Term $\ln \left(\frac{p_{i g}}{p_{i \infty}}\right)$ relates to the change in the monomer concentration near the particle surface and it is described by the following equation (Nadykto et al., 2003)

$\ln \left(\frac{p_{i g}}{p_{i \infty}}\right)=\frac{\alpha_{i}\left(q e_{0}\right)^{2}}{32 \pi^{2} \varepsilon_{0}^{2} r^{4} k T}$
$+\ln \left[\frac{\sinh \left(\frac{q e_{0} l_{i}}{4 \pi \varepsilon_{0} k T r^{2}}\right)}{\frac{q e_{0} l_{i}}{4 \pi \varepsilon_{0} k T r^{2}}}\right]=C_{i}(r, l, T)$.

where $\sinh (z)=[\exp (z)-\exp (-z)] / 2, l_{i}$ is the dipole moment of component $i$ and $\alpha_{i}$ is the polarizability of component $i$ and $C_{i}(r, l, T)$ is the correction to the condensing vapour pressure due to the dipole-charge interaction. Now we insert the expression for the change in chemical potentials (5-6) into Eq. (3), and get the analytical expression for the change in the Gibbs free energy

$$
\begin{aligned}
& \Delta G=-k T\left[\ln \left(\frac{A_{a g \infty}}{A_{a L}}\right) n_{a}+\ln \left(\frac{A_{b g \infty}}{A_{b L}}\right) n_{b}\right] \\
& -k T\left[C_{a} n_{a}+C_{b} n_{b}\right]+\sigma A(r) \\
& +\frac{q^{2} e_{o}^{2}}{8 \pi \varepsilon_{0}}\left[\frac{1}{\varepsilon_{g}}-\frac{1}{\varepsilon_{r}}\right]\left[\frac{1}{r}-\frac{1}{r_{0}}\right]
\end{aligned}
$$

Applying the Gibbs-Duhem identity (Renninger et al., 1981) to function $\Delta G$ at constant temperature and pressure,

$d \Delta G=0$,

we obtain, after differentiation with rearranging the terms, the following set

$$
\begin{aligned}
& -k T\left(\ln \left(\frac{A_{a g \infty}}{A_{a L}}\right)+C_{a}\right) \\
& +\frac{m_{a}}{\rho}\left[\left(\frac{2 \sigma}{r}\right)-\frac{q^{2} e_{o}^{2}}{32 \pi^{2} \varepsilon_{0}}\left[\frac{1}{\varepsilon_{g}}-\frac{1}{\varepsilon_{r}}\right]\left[\frac{1}{r^{4}}\right]\right]=0, \\
& -k T\left(\ln \left(\frac{A_{b g \infty}}{A_{b L}}\right)+C_{b}\right) \\
& +\frac{m_{b}}{\rho}\left[\left(\frac{2 \sigma}{r}\right)-\frac{q^{2} e_{o}^{2}}{32 \pi^{2} \varepsilon_{0}}\left[\frac{1}{\varepsilon_{g}}-\frac{1}{\varepsilon_{r}}\right]\left[\frac{1}{r^{4}}\right]\right]=0,
\end{aligned}
$$




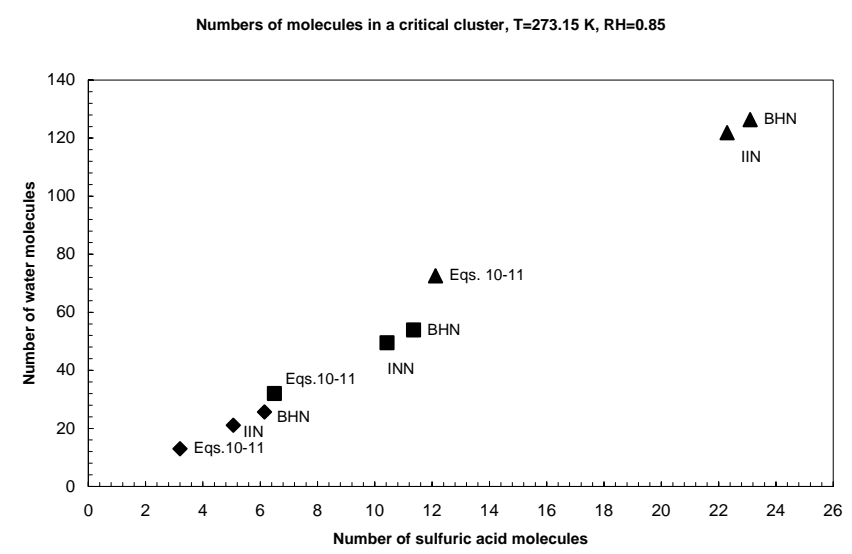

Fig. 1. Numbers of molecules in critical cluster as a function of temperature, relative humidity and sulfuric acid concentration. Triangles correspond to the sulfuric acid concentration of $10^{7}$, squares to that of $10^{8}$ and diamonds to that of $10^{9} \mathrm{~cm}^{-3}$. T $=273.15 \mathrm{~K}$, $\mathrm{RH}=0.85$.

Now we multiply sides of Eqs. (8) and (9) by $m_{a}^{-1}$ and $-m_{b}^{-1}$ respectively and sum the equations obtained to get

$\frac{\left(\ln \left(\frac{A_{a g \infty}}{A_{a L}}\right)+C_{a}\right)}{\left(\ln \left(\frac{A_{b g \infty}}{A_{b L}}\right)+C_{b}\right)}=\frac{v_{a}}{v_{b}}$,

In prevailing temperature and saturation ratios of the components $a$ and $b$, solution to the set of Eqs. $(10,11)$ or $(9$, 11) gives us the numbers of molecules of components $a$ and $b$ in the cluster and the corresponding the cluster size. In the case of non-polar vapours $l_{a} \rightarrow 0, l_{b} \rightarrow 0$ set (10-11) reduces to Eqs. (1)-(2) predicting the composition and size of the critical cluster in the classical IIN theory. The derived model can be considered as a generalization of the classical binary IIN theory because it not only accounts for all the mechanisms involved in classical binary IIN but also include the effect of the dipole -charge interaction neglected in the classical binary IIN theory.

Since the present model is derived, as well as the classical IIN theory, assuming the bulk surface tension, density and dielectric constants of the condensed matter, it might be generally limited when the critical cluster is composed of $n \ll 1000$ molecules. The quality of the measurements of the bulk surface tension applied in the nucleation models is another important issue. It is well known that the values of the surface tension given by different methods such as maximum bubble pressure, capillary rise and Wilhelmi plate (or other contact methods) often deviates by several dynes. Since the thermodynamics of the cluster formation depends strongly on the surface tension of the substance nucleating, in the case of disagreement between theoretical predictions and experimental data it is difficult to figure out whether the capillary approximation is imperfect or the bulk surface tension is measured inaccurately.

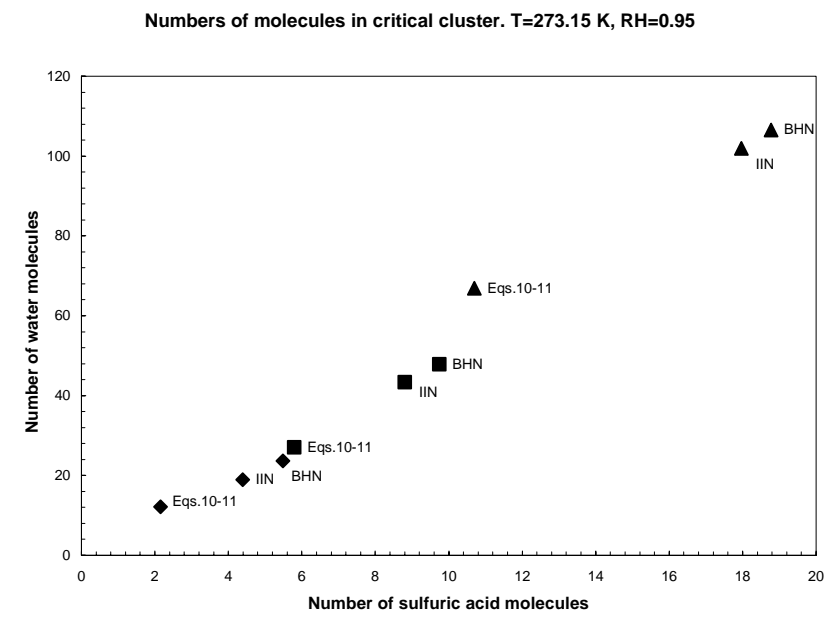

Fig. 2. Numbers of molecules in critical cluster as a function of temperature, relative humidity and sulfuric acid concentration. Triangles correspond to the sulfuric acid concentration of $10^{7}$, squares to that of $10^{8}$ and diamonds to that of $10^{9} \mathrm{~cm}^{-3}$. T $=273.15 \mathrm{~K}$, $\mathrm{RH}=0.95$.

Although the present theory is not focused on the sign effect, it possesses some potential for explaining the sign preference because both the enhancement factor for the condensation/nucleation rates (Nadykto and Yu, 2003) and correction to the chemical potential of the condensable vapour molecules due to the dipole-charge interaction strongly depend on the stretch of electrical field and the mean cluster density, which may be different for positive and negative ions due to different geometry and charge distribution. In order to study the sigh effect quantitatively, the detailed information about structure and properties of the cluster ions have to be obtained.

\section{Results and Discussion}

Calculations were performed using Eqs. (10)-(11) for binary sulfuric acid-water vapour mixture. Binary clusters are singly charged. Values of input parameters have been adopted from CRC Handbook of Chemistry and Physics (2002), Kulmala et al. (1998) and Myhre et al. (1998). Figures 1-4 show the comparisons of the cluster sizes as functions of ambient temperature $(\mathrm{T})$, relative humidity $(\mathrm{RH})$, and the concentration of sulfuric acid vapour calculated from Eqs. (1) - (2) $q=1$ (IIN) and $q=0$ (BHN, binary homogeneous nucleation), and Eqs. (10)-(11) (this study).

As may be seen from Figs. 1-4, dipole-charge interaction significantly influences the formation of small cluster ions, reducing the number of molecules in the critical cluster and, consequently, decreasing the critical size. Difference between results given by the considered theories rises as the cluster size decreases. For small clusters, the difference in the numbers of molecules in the critical cluster may be as 


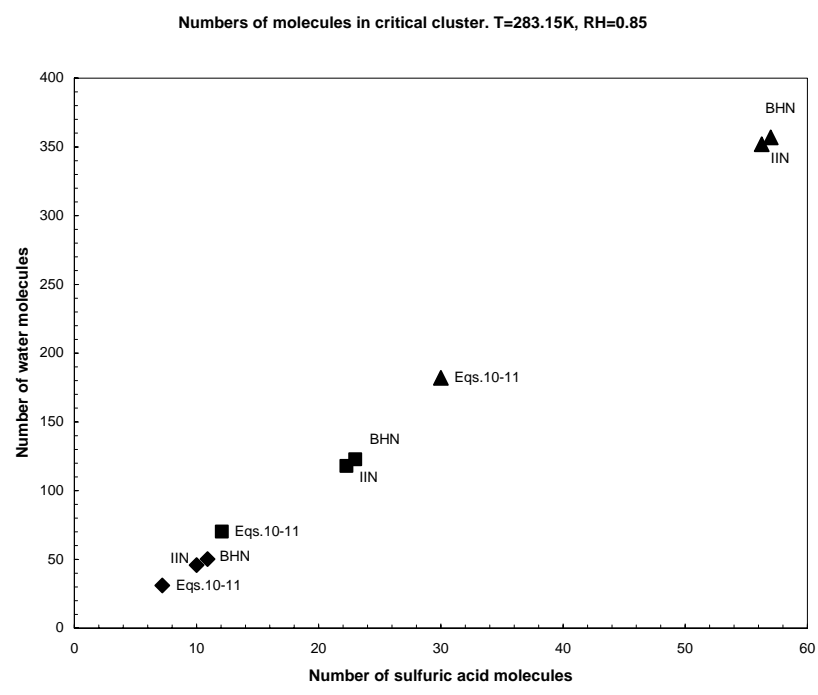

Fig. 3. Numbers of molecules in critical cluster as a function of temperature, relative humidity and sulfuric acid concentration. Triangles correspond to the sulfuric acid concentration of $10^{7}$, squares to that of $10^{8}$ and diamonds to that of $10^{9} \mathrm{~cm}^{-3}$. $\mathrm{T}=283.15 \mathrm{~K}$, $\mathrm{RH}=0.85$.

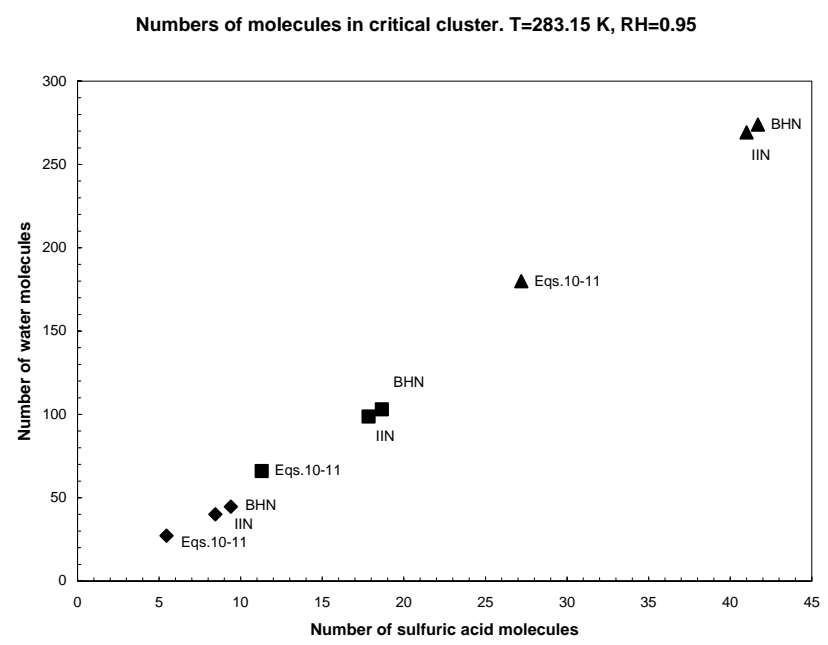

Fig. 4. Numbers of molecules in critical cluster as a function of temperature, relative humidity and sulfuric acid concentration. Triangles correspond to the sulfuric acid concentration of $10^{7}$, squares to that of $10^{8}$ and diamonds to that of $10^{9} \mathrm{~cm}^{-3}$. T $=283.15 \mathrm{~K}$, $\mathrm{RH}=0.95$.

big as more than 2 times. The deviation between IIN theory and present theory rises when the relative humidity and sulfuric acid concentration are growing. The contribution of the Thomson effect is smaller than that of the dipole-charge interaction that is essential for the nucleation from highly polar vapours. As may be seen from Figs. 1-4, the classical Kelvin-Thomson equation significantly overestimates the number of the molecules in the critical cluster compared to

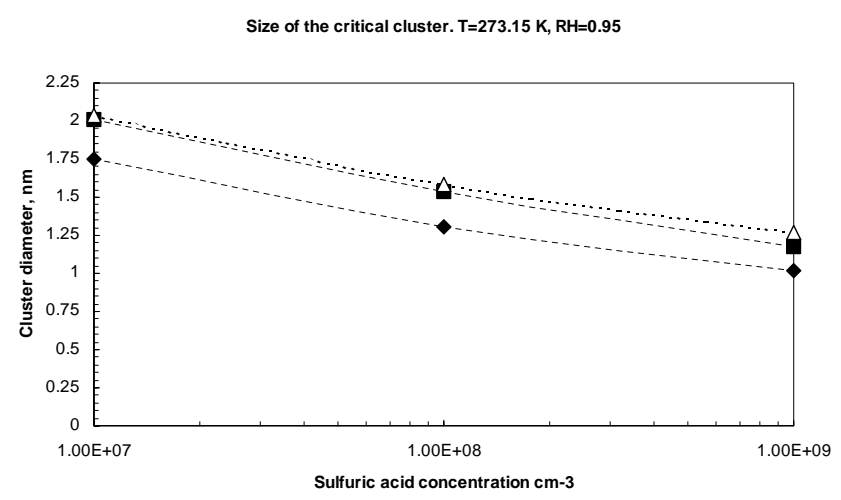

Fig. 5. Size of the critical cluster given by different models. Triangles correspond to BHN, squares to classical IIN and diamonds present the results of the present study. $\mathrm{T}=273.15 \mathrm{~K}, \mathrm{RH}=0.95$.

results predicted by the present theory in all the cases studied here. Since both the sulfuric acid ( $l=2.72$ Debyes) and water ( $l=1.85$ Debyes) are highly polar, such a big effect of the dipole charge-interaction is not surprising. We would like to emphasize that the domination of the effect related to the dipole-charge interaction over the Kelvin-Thomson effect is not a must. This effect is essential for polar gases only.

To illustrate the consequences of Eqs. (10)-(11) in terms of the cluster size and thermodynamics of the cluster formation, we calculated the critical cluster sizes (Fig. 5). As seen from Fig. 5, the deviation between the classical IIN theory and the present study is $\sim 15$ percent. The complete theory of the nucleation rates requires corrections to both the Gibbs free energy and forward (condensation) rate to be accounted for simultaneously. The contribution of the dipole-charge interaction to the growth kinetics may be significant (Nadykto and $\mathrm{Yu}, 2003$ ) and, thus, the derivation of the nucleation rates is not as straightforward as it could be expected. The work on the model of the nucleation rates is in progress and we plan to publish it elsewhere.

\section{Summary}

In this paper we developed the model of ion-induced nucleation of two-component polar vapours. It has been shown that the formation of small ion clusters is influenced by the vapour polarity and the dipole-charge interaction decreases the size of critical clusters formed. It has been demonstrated that the actual size of small binary ion clusters may deviate significantly from the size predicted by classical KelvinThomson theory, when the highly polar vapours are nucleating. The derived model can be considered as a generalized reformulation of the classical IIN theory extended to the nucleation in polar vapours. Based on the results obtained we suggest that the dipole moment of the condensing monomers is likely to be a new parameter controlling the binary ioninduced nucleation. 
Acknowledgements. This work was supported by the NSF under grant ATM 0104966.

Edited by: T. Röckmann

\section{References}

Arnold, F.: Multi-ion complexes in the stratosphere - Implications for trace gases and aerosol, Nature, 284, 610-611, 1980.

Carslaw, K. S., Harrison, R. G., and Kirkby, J.: Cosmic rays, clouds, and climate, Science, 298, 1732-1737, 2002.

Castleman Jr., A. W., Holland, P. M., and Keesee, R. G.: The properties of ion clusters and their relationship to heteromolecular nucleation, J. Chem. Phys., 68, 1760-1767, 1978.

CRC handbook of chemistry and physics: Cleveland, Ohio, CRC Press, 2002.

Eichkorn, S., Wilhelm, S., Aufmhoff, H., Wohlfrom, K. H., and Arnold, F.: Cosmic ray-induced aerosol-formation: First observational evidence from aircraft-based ion mass spectrometer measurements in the upper troposphere, Geophys. Res. Lett., 29(14), 1698, doi:10.1029/2002GL015044, 2002.

Hamill, P., Turco, R. P., Kiang, C. S., Toon, O. B., and Whitten, R. C.: An analysis of various nucleation mechanisms for sulfate particles in the stratosphere, J. Aerosol Sci., 13, 561-585, 1982.

Jaecker-Voirol, A. and Mirabel, P.: Heteromolecular nucleation in the sulfuric acid-water system, Atmos. Environ., 23, 2053-2057, 1989.

Korshunov, V. K.: Ravnovesie para s zaryiazennoy kaplei, Izvestia AN USSR, ser. Phys.Atm. \& Ocean, 92-94, 1980.

Kulmala, M., Laaksonen, A., and Pirjola, L.: Parameterizations for sulfuric acid/water nucleation rates, J. Geophys. Res., 103, 83018307, 1998.
Laakso, L., Makela, J., Pirjola, L., and Kulmala, M.: Model studies on ion-induced nucleation in the atmosphere, J. Geophys. Res., 107, 10.1029/2002JD002140, 2002.

Mohnen, V. A.: Discussion of the formation of major positive and negative ions up to the $50 \mathrm{~km}$ level, Pure Appl. Geophys., 84, 141-153, 1971.

Myhre, C. E. L., Nielsen C. J., Saastad, O. W.: Density and surface tension of aqueous $\mathrm{H}_{2} \mathrm{SO}_{4}$ at low temperature, J. Chem. Eng. Data, 43, 617-622, 1998.

Nadykto, A. B., Mäkelä, J., Yu, F., Kulmala, M., and Laaksonen, A.: Comparison of the experimental mobility equivalent diameter for small cluster ions with theoretical particle diameter corrected by effect of vapour polarity, Chem. Phys. Lett., 382/1-2, 6-11, 2003.

Nadykto A. B. and Yu, F.: Uptake of neutral polar vapour molecules by charged particles: Enhancement due to dipole-charge interaction, J. Geophy. Res., 108(D23), 4717, doi:10.1029/2003JD003664, 2003.

Raes, F., Augustin, J., and Vandingenen, R.: The role of ion-induced aerosol formation in the lower atmosphere, J. Aerosol Sci., 17, 466-470, 1986.

Renninger, R. G., Hiller, F. C., and Bone, R. C.: Comment on "Selfnucleation in the sulfuric acid-water system", J. Chem. Phys., 75, 1584-1585, 1981.

Yu, F.: Altitude variations of cosmic ray induced production of aerosols: Implications for global cloudiness and climate, J. Geophy. Res., 107(A7), 10.1029/2001JA000248, 2002.

Yu, F.: Nucleation rate of particles in the lower atmosphere: Estimated time needed to reach pseudo-steady state and sensitivity to $\mathrm{H}_{2} \mathrm{SO}_{4}$ gas concentration, Geophys. Res. Lett., 30, 1526, doi:10.1029/2003GL01708, 2003.

Yu, F. and Turco, R. P.: Ultrafine aerosol formation via ionmediated nucleation, Geophys. Res. Lett., 27, 88-886, 2000. 